\title{
Clinical Characteristics of Pediatric Bipolar Disorder by Subtype in a Korean Inpatient Sample
}

\author{
Subin Park, M.D., Ph.D. ${ }^{1)}$, Soo-Churl Cho, M.D., Ph.D. ${ }^{2)}$, Ohyang Kwon, M.D. ${ }^{3)}$, \\ Jeong-Hoon Bae, M.D., Ph.D. ${ }^{4)}$, Jae-Won Kim, M.D., Ph.D. ${ }^{2)}$, Min-Sup Shin, Ph.D. ${ }^{2}$, \\ Hee-Jeong Yoo, M.D., Ph.D. ${ }^{2)}$, and Bung-Nyun Kim, M.D., Ph.D. ${ }^{2)}$

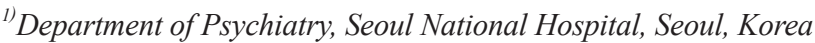 \\ ${ }^{2)}$ Department of Psychiatry and Behavioral Science, Seoul National University College of Medicine, Seoul, Korea \\ ${ }^{3)}$ Department of Psychiatry, Hyewon St. Mary's Hospital, Chilgok, Korea \\ ${ }^{4)}$ Department of Neuropsychiatry, Bongseng Memorial Hospital, Busan, Korea
}

\begin{abstract}
Objectives : We compared the clinical presentations of manic and depressive episodes and the treatment response among children and adolescents with bipolar disorder (BD) types I and II and BD not otherwise specified (NOS).

Methods : The sample consisted of 66 patients, aged between 6 and 18 years, who were admitted for BD to a 20-bed child and adolescent psychiatric ward in a university hospital located in Seoul, Korea.

Results : Patients with BD type I were more likely to have lower intelligence quotients and exhibit violent behaviors during manic episodes than patients with BD type II or BD NOS and to show better treatment responses during manic episodes than patients with BD NOS. Patients with BD NOS were more likely to have an irritable mood rather than a euphoric mood during the manic phase than patients with BD type I or II and to exhibit violent behaviors during the depressive phase and chronic course than patients with BD type II.
\end{abstract}

Conclusion : Pediatric BD patients are heterogeneous with respect to their clinical characteristics. Implications for the usefulness of the current diagnostic subtype categories should be investigated in future studies.

KEY WORDS : Bipolar Disorder · Child and Adolescent · Clinical Characteristics · Subtype.

\section{Introduction}

The Diagnostic and Statistical Manual of Mental Disorders, Fourth Edition (DSM-IV) classifies bipolar disorder (BD) into four clinical subtypes, BD type I (BD I: manic or mixed episodes associated with major depressive episodes), BD type II (BD II: depressive episodes accompanied by hypomanic, but not manic episodes), cyclothymic disorders with hypomanic episodes associated with depressive symptoms that do not meet the criteria for a major depressive episode, and BD not otherwise specified (BD NOS, with bipolar features that do not fully meet the diagnostic criteria of type I or

Date received : April 27, 2015

Date of revision : June 4, 2015

Date accepted : June 5, 2015

Address for correspondence : Bung-Nyun Kim, M.D., Ph.D., Department of Psychiatry and Behavioral Science, Seoul National University College of Medicine, 101 Daehak-ro, Jongno-gu, Seoul 03080, Korea

Tel : +82.2-2072-3647, Fax : +82.2-747-5774

E-mail : kbn1@snu.ac.kr

This work was supported by the National Research Foundation of Korea (NRF) Grant funded by the Korean Govrnment (MSIP) (NRF2014R1A1A3049818). type II). ${ }^{1)}$ In the past decade, a heated debate has emerged regarding whether these subtypes are separate disorders or are part of a single spectrum. ${ }^{2)}$ Diagnosing BD and DSM-IV subtype is more difficult in children and adolescents than in adult populations due to their variability in clinical presentation, high comorbidity with other psychiatric disorders, and the effects of development on symptom expression. ${ }^{3-6)}$

A National Institute of Mental Health committee ${ }^{7)}$ proposed differentiation between a narrow and a broad phenotype, in accordance with individual patient fit to the full DSM-IV criteria. The committee addressed the complex problem of BD NOS, including in this subtype in patients with manic episodes shorter than 4 days, those presenting with fewer (by at least one) manic symptoms than the B criteria, those presenting with an irritable mood and sufficient symptoms but a chronic course, or patients with manic symptoms evident only at home. An excellent consensus was exhibited only for the narrow phenotype, whereas much poorer agreement was exhibited for the BD NOS subtype. Despite this, the DSM-IV categorization of BD has been applied to children and adolescents-as it has to the adult population- 
and BD NOS is more commonly diagnosed in children and adolescents with bipolar symptoms than in adults due to the frequent atypicality in the presentation and course of pediatric $\mathrm{BD}$. Therefore, greater understanding of the clinical implications and presentations of BD NOS are needed to clarify the diagnosis of pediatric $\mathrm{BD}$.

In Diagnostic and Statistical Manual of Mental Disorders, Fifth Edition (DSM-5), ${ }^{8)}$ the diagnostic criteria of BD I and BD II are similar to those of the DSM-IV, but some minor changes were made. To enhance the accuracy of diagnoses and facilitate earlier detection in clinical settings, the primary criteria for manic and hypomanic episodes (criterion A) now includes an emphasis on changes in activity and energy-not just mood. The DSM-IV diagnosis of BD I, mixed episode, requiring that the individual simultaneously meet the full criteria for both manic episodes and major depressive episodes, has been removed from the DSM-5. Instead, a new specifier, "with mixed features", has been added, that can be applied to the current manic, hypomanic, or depressive episodes in BD I or BD II. The DSM-5 has replaced the DSM-IV's vague BD NOS category by the specification of particular conditions for other specified bipolar and related disorder, including major depressive episode with the subthreshold conditions of bipolarity, for instance, allowing a duration of 2 to 3 days for hypomanic episodes or fewer than four symptoms of hypomania during 4 days, hypomanic episodes without major depressive episodes, and short-duration cyclothymia (less than 24 months).

Most studies of pediatric BD include only BD I or do not differentiate BD I, BD II, and BD NOS. To date, we know of only two studies that directly compare the clinical features of juvenile BD according to DSM-IV subtype. In a study of 217 bipolar children and adolescents in a single research center in Italy, Masi et al. ${ }^{9)}$ reported that patients with BD NOS presented with earlier onset of the disorder, a chronic rather than an episodic course, an irritable rather than an elated mood, and a more frequent lifetime comorbidity with attention-deficit hyperactivity disorder (ADHD) and oppositional defiant disorder (ODD) than patients with BD I or BD II. Birmaher et al. ${ }^{10)}$ reported on a large sample of 263 bipolar children and adolescents recruited in a multisite study (courses and outcomes of bipolar illness in youth). They found that the frequency of ADHD was similar in BD I and BD NOS (61.2\% and $58.8 \%$, respectively), whereas ODD and anxiety disorders did not differentiate BD I, BD II, and BD NOS. Patients with BD NOS showed an earlier age of onset, required a longer time to recover, and spent more time with subsyndromal mania and/or mixed symptoms, but had a longer recurrence time than patients with BD I or BD II. However, these results are limited because the cited studies did not differentiate manic and depressive episodes when comparing clinical features.

In light of this paucity of studies of the implications of subtyping in patients with pediatric $\mathrm{BD}$, we compared premorbid features, clinical presentations, and treatment responses among children and adolescents with BD I, II, and NOS.

\section{Methods}

\section{Subjects and procedures}

Patients were diagnosed with bipolar affective disorder based upon the DSM-IV, Text Revised. ${ }^{11)}$ All patients admitted to a 20-bed child and adolescent psychiatric ward in a university hospital located in Seoul, Korea from 2007 to 2011 were identified from electronic medical records and included in the study. Our psychiatric ward is one of two closed psychiatric wards exclusively for child and adolescent patients in Korea. Standard medical record requirements for the participating hospital included one primary diagnosis and several auxiliary diagnoses in a discharge summary, which had been recorded by an attending psychiatrist after close inpatient observation, neuropsychological testing, and a clinical interview. Nurse coordinators identified eligible patients who had primary diagnoses of 'bipolar affective disorder' through reviews of admission patient lists. A board-certified child and adolescent psychiatrist reviewed the subjects' electronic medical records and ascertained psychiatric diagnoses. In our study, we defined BD NOS according to DSM-5 criteria for other specified bipolar and related disorder (details presented in the Introduction). The $\mathrm{k}$ value for subtyping between the reviewing psychiatrist and each of the psychiatrists who had recorded the diagnosis was .89. The average length of stay was approximately 6 weeks, the number of admission of each patient ranged from 1 to 4 , and the rates of recurrent admission (admission more than two times during a 5-years period) was $20 \%$. For patients who had recurrent admissions, the medical records of each admission were reviewed.

Psychiatric diagnoses preceding the $\mathrm{BD}$ diagnosis and the onset age were investigated. The characteristics of episodes at admission (age at admission, duration between recent onset and admission, duration of admission, and comorbidity) were also examined. We indexed (hypo)manic episodes and depressive episodes separately for each patient. Episodes at admission were of primary importance, and if the patient was admitted for a hypo(manic) episode, the most recent depressive episode was used as an index for the depressive episode, 
or vice versa. For patients who had recurrent admissions, the most recent admission was chosen as an index episode. The symptom characteristics (prevalent mood, violent behaviors, and psychotic symptoms) and treatment response for each index episode were evaluated. Patients with scores of 1 (very much improved) or 2 (much improved) on the CGI-I at the time of discharge were classified as good treatment responder group whereas subjects with scores of 3-7 deemed poor responder group.

In addition, we obtained information regarding patient intelligence quotient (IQ), any family history of BD or other psychiatric disorders, and the past clinical course of BD (episodic versus chronic).

All of the above information was obtained from a boardcertified psychiatrist and from each of the psychiatrists who had been an attending psychiatrist for each patient via a retrospective chart review. All information was recorded in the admission notes by an attending psychiatric resident. Patient IQ was measured by a psychologist using the Korean Educational Development Institute Wechsler Intelligence Scale for Children-Revised at the beginning of the admission. ${ }^{12)}$ Family histories of $\mathrm{BD}$ or other psychiatric disorders were obtained from the parent report. When determining whether the clinical course was episodic or chronic the following criteria were used : episodes had to last at least 7 days with marked worsening of the clinical course, and any remission of the episodes was defined as a return to baseline with a symptom free interval of at least 1 month. Patients who did not show clearly defined episodes or patients in their first episode who did not return to baseline were considered to present a chronic course.

The study was approved by the Institutional Review Board for human subjects at the Seoul National University Hospital.

\section{Statistical analysis}

Descriptive statistics for the sample characteristics are presented. We compared the clinical differences between patients with BD I, BD II, and BD NOS using analysis of variance for the continuous variables and chi-squared tests or binary logistic regression analyses for categorical variables. All statistical analyses were performed using SPSS (version 21.0; SPSS Inc., Chicago, IL, USA), and statistical significance was defined as an alpha level <.05.

\section{Results}

A total of 66 patients ( 36 boys and 30 girls), aged between 6 and 18 years, were enrolled in the study. The mean age was 14.0 (standard deviation 1.7) years. Of the patients in the study,

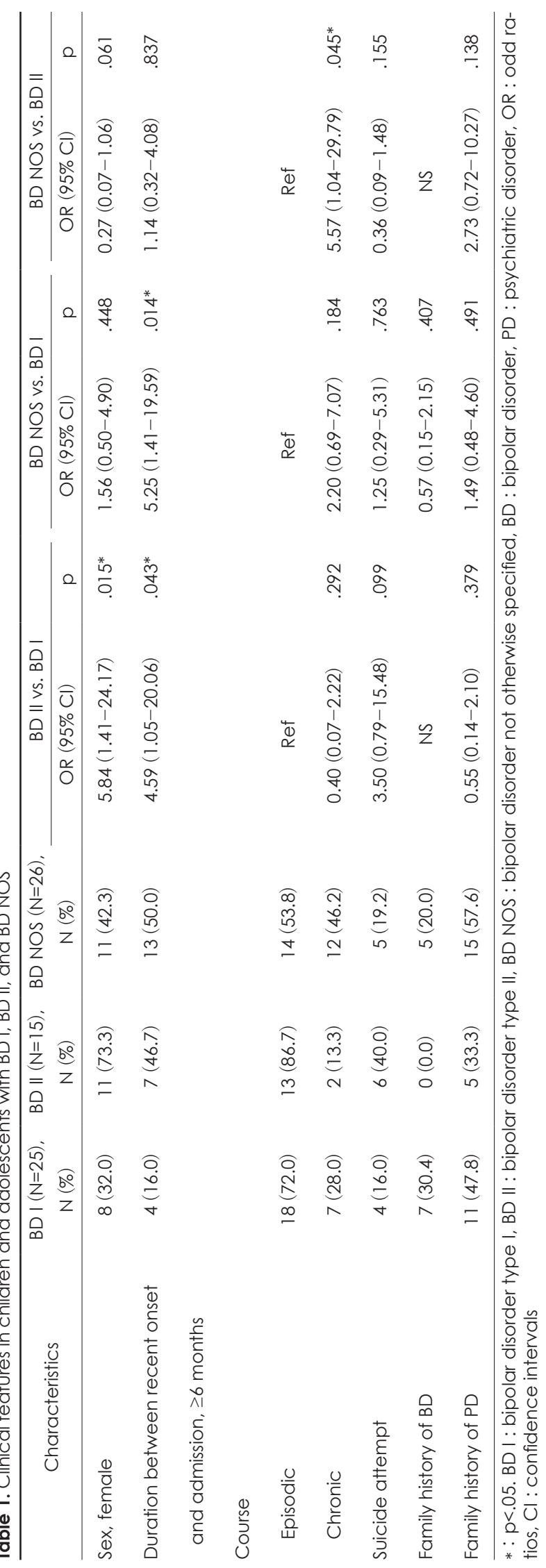


25 were diagnosed with BD I, 15 were diagnosed with BD II, and 26 were diagnosed with BD NOS.

Tables 1 and 2 show the difference in clinical features according to the subtype of BD. Patients with BD II were more likely to be female than patients with BD I, and were older at admission than patients with BD I or BD NOS. Both patients with BD II and BD NOS had higher IQs, in particular higher performance IQs, than patients with BD I. Patients with BD NOS more frequently showed a chronic course than those with BD II.

The most common current comorbid disorders were ADHD (28.0\%) for BD I, conduct disorder (CD)/ODD (20.0\%) for BD II, and ADHD (26.9\%) and CD/ODD (23.1\%) for BD NOS (Fig. 1).

Table 3 shows the clinical manifestations of the manic phase in children and adolescents with BD I, BD II, and BD NOS. Patients with BD NOS were more likely to have an irritable/dysphoric mood rather than a euphoric mood during the (hypo)manic phase than patients with BD I or BD II. Patients with BD I were more likely to present with violent behaviors during manic/mixed episodes than those with BD II or BD NOS. The treatment response during the manic phase was the best among patients with BD I and the worst among patients with BD NOS.

Table 4 shows the clinical manifestations of the depressive phase in children and adolescents with BD I, BD II, and BD NOS. Patients with BD NOS were more likely to present with an irritable mood and violent behaviors during the depressive episode than those with BD II. There was no difference in the treatment response during the depressive phase between the groups.

\section{Discussion}

The major findings of this study are as follows: 1) patients with BD I are more likely to have lower IQs and present with violent behaviors during manic episodes than those with $\mathrm{BD}$
II or BD NOS, 2) patients with BD II are older at admission and are more likely to be female than patients with BD I, and 3) patients with BD NOS are more likely to have an irritable mood rather than a euphoric mood during the manic phase than those with BD I or BD II and show less treatment response during the manic phase than those with BD I, and they are more likely to present violent behaviors during the depressive phase and chronic course than BD II patients.

Previous studies have reported a more chronic course for BD NOS than for BD I or BD II. ${ }^{9,10)}$ Our result also showed a more chronic course for BD NOS. The older age at onset of mood disorder and at admission in the patients with BD II was consistent with previous studies. ${ }^{910)}$ This result could be due to a lesser disease severity or to more difficulty in the detection of a hypomanic episode than a manic/mixed episode.

Previous studies have indicated that adults with BD have minimal to no impairments on standard measures of intellectual functioning, suggesting that the cognitive deficit profile in $\mathrm{BD}$ does not involve gross intellectual decline. ${ }^{13)}$ Metaanalysis of the existing literature comparing pediatric cases with BD to healthy controls also indicated little difference in the overall intellectual ability of individuals with BD and controls. ${ }^{14)}$ In our study, though the patients were in the symptomatic phase during admission, patients with $\mathrm{BD}$ II and $\mathrm{BD}$ NOS showed no impairments on standard measures of intellectual functioning. In contrast, patients with BD I showed lower IQs, in particular lower performance IQs, than patients with BD II or BD NOS. While there are a limited number of adult studies that have attempted to differentiate cognitive profiles according to the subtype of $\mathrm{BD}$, to our knowledge, there was only one study that investigated neurocognitive differences between BD I and BD II in pediatric samples. ${ }^{15)}$ In the cited study, BD I patients are characterized by more severe cognitive impairment relative to BD II patients who show an intermediate pattern of performance between BD I patients and healthy controls. ${ }^{15)}$ Further studies are needed to identify whether these cognitive characteristics of BD I are

Table 2. Comparison of age and $I Q$ according to subtypes of $B D$

\begin{tabular}{|c|c|c|c|c|c|c|c|}
\hline Characteristics & $\begin{array}{c}\mathrm{BD} \mathrm{l}^{\mathrm{a}}(\mathrm{N}=25) \\
\text { mean }(\mathrm{SD})\end{array}$ & $\begin{array}{c}\mathrm{BD} \|^{\mathrm{b}}(\mathrm{N}=15), \\
\text { mean }(\mathrm{SD})\end{array}$ & $\begin{array}{c}\mathrm{BD} \mathrm{NOS}^{\mathrm{C}}(\mathrm{N}=26) \\
\text { mean }(\mathrm{SD})\end{array}$ & $\mathrm{F}$ & $p$ & $\eta^{2}$ & Contrast \\
\hline Age at admission & $13.3(2.5)$ & $15.2(1.9)$ & $13.3(2.5)$ & 3.78 & $.028^{*}$ & 0.11 & $b>a, c$ \\
\hline Age at first diagnosis of $\mathrm{BD}$ & $13.2(2.5)$ & $14.9(2.1)$ & $12.9(2.4)$ & 2.96 & .059 & 0.09 & \\
\hline Age at first diagnosis of PD & $11.2(3.5)$ & $13.3(2.9)$ & $11.3(3.4)$ & 2.13 & .128 & 0.06 & \\
\hline Full scale IQ & $89.8(19.3)$ & $105.1(15.6)$ & $103.4(13.5)$ & -2.0 & $.049 *$ & 0.16 & $a<b, c$ \\
\hline Verbal IQ & $94.8(16.6)$ & $102.5(14.2)$ & $100.5(12.1)$ & 1.5 & .242 & 0.05 & \\
\hline Performance IQ & $86.4(21.4)$ & $106.7(16.7)$ & $103.1(13.5)$ & 7.5 & $.001^{\dagger}$ & 0.21 & $a<b, c$ \\
\hline
\end{tabular}

$*: p<.05,{ }^{\dagger}: p<.01 . \mathrm{BD}$ I : bipolar disorder type I, BD II : bipolar disorder type II, BD NOS : bipolar disorder not otherwise specified, $\mathrm{BD}$ : bipolar disorder, $\mathrm{PD}$ : psychiatric disorder, $\mathrm{IQ}$ : intelligence quotient, $\mathrm{SD}$ : standard deviation 
restricted to the symptomatic phase.

During the (hypo)manic phase, the prevalent mood was elated in BD I and BD II patients but irritable in BD NOS patients. Furthermore, patients with BD I had a higher frequency of violent behaviors than those with BD II or BD NOS. These results are consistent with previous studies. ${ }^{9,10)}$ However, previous studies did not consider in which phase the violent symptoms occurred. Our finding of less treatment response during the manic phase in patients with BD NOS than those with BD I corresponds to the results of a previous longitudinal follow-up study, which indicated that subjects with BD NOS took approximately three times as long to recover from their index mood episode than those with BD I, and they spent fewer weeks free of clinically significant mood symptoms during a prospective follow-up. ${ }^{10)}$ During the depressive phase, patients with BD NOS had a higher frequency of violent behaviors than those with BD II. This result may be explained by the fact that BD NOS patients showed a trend toward a higher frequency of irritable mood rather than depressed mood.

Birmaher et al. ${ }^{10)}$ proposed a lifetime history of comorbid disorders more commonly including ADHD for BD I, any anxiety disorder and ODD for BD II, and ADHD and ODD for BD NOS. Their findings are consistent with ours with respect to the high current comorbidity of ADHD for BD I, ODD, and anxiety disorder for BD II, and ADHD and ODD for BD NOS, although the frequency of comorbidity was approximately two times higher in the previous study than in our study. This discrepancy likely occurred because Birmaher et al. reported lifetime comorbidity rates, whereas we reported current comorbidity rates. Children with BD have a high rate of comorbid ADHD, ${ }^{16,17)}$ and some researchers suggest that children presenting with ADHD often have an occult mood disorder, especially unrecognized bipolarity. ${ }^{18,19)}$ Symptom overlap between BD and ADHD, such as hyperactivity, accelerated speech, and distractibility, make differential diagnosis a challenge.

The present study has several limitations. First, the study employed a retrospective methodological design. Because data were gathered from electronic medical charts rather than face-to-face interviews, some important information-such as the severity of the mood episodes or the number of previous episodes-was not available in all patients and therefore was not included as a potential correlate variable in this study. Second, because the study was conducted in a single university hospital, the findings may not be representative of all psychiatric adolescent inpatients in Korea. Third, the medication history of patients was not included in this study. Finally, our small sample size may have reduced the likelihood of finding statistical significance. Thus, additional prospective, controlled studies in many hospitals nationwide are required.

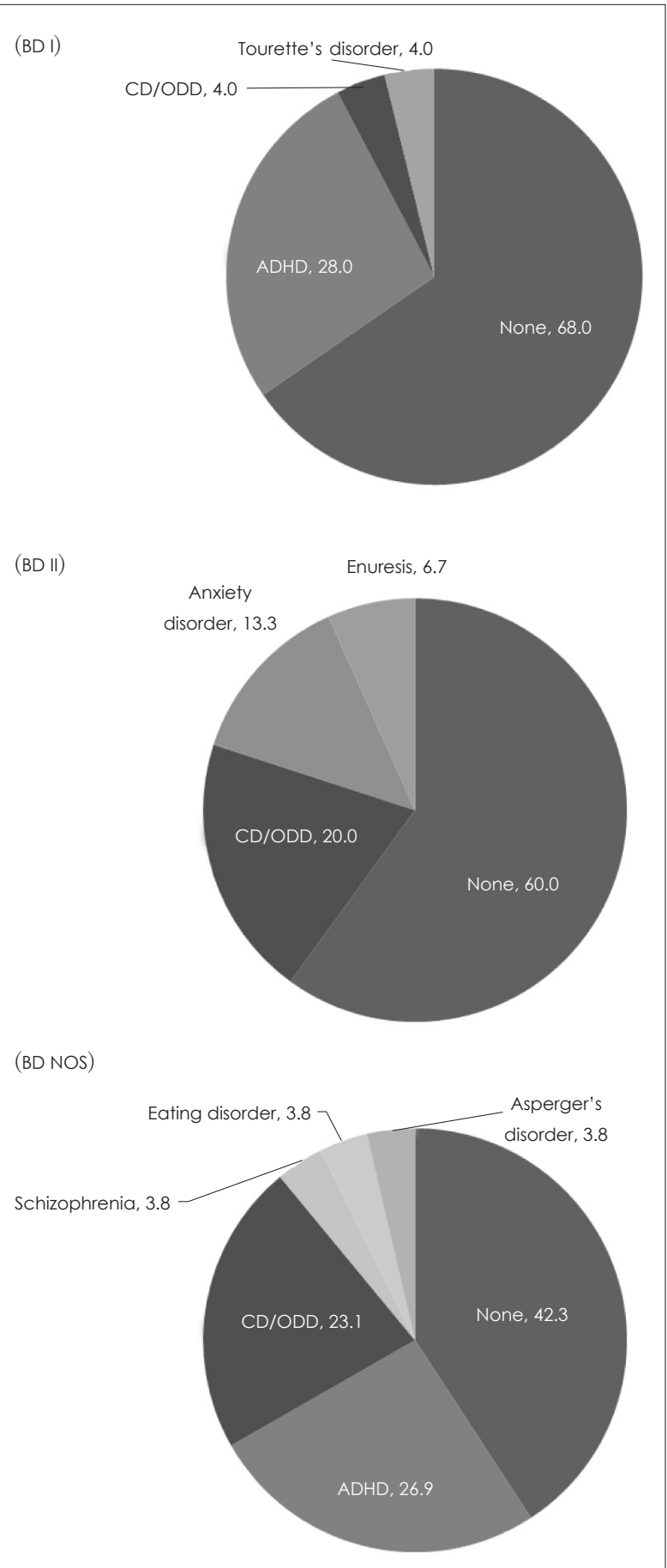

Fig. 1. Current comorbid disorders in children and adolescents with BD I, BD II, and BD NOS. BD I : bipolar disorder type I, BD II : bipolar disorder type II, BD NOS : bipolar disorder not otherwise specified, ADHD : attention-deficit hyperactivity disorder, CD/ ODD : conduct disorder/oppositional defiant disorder 


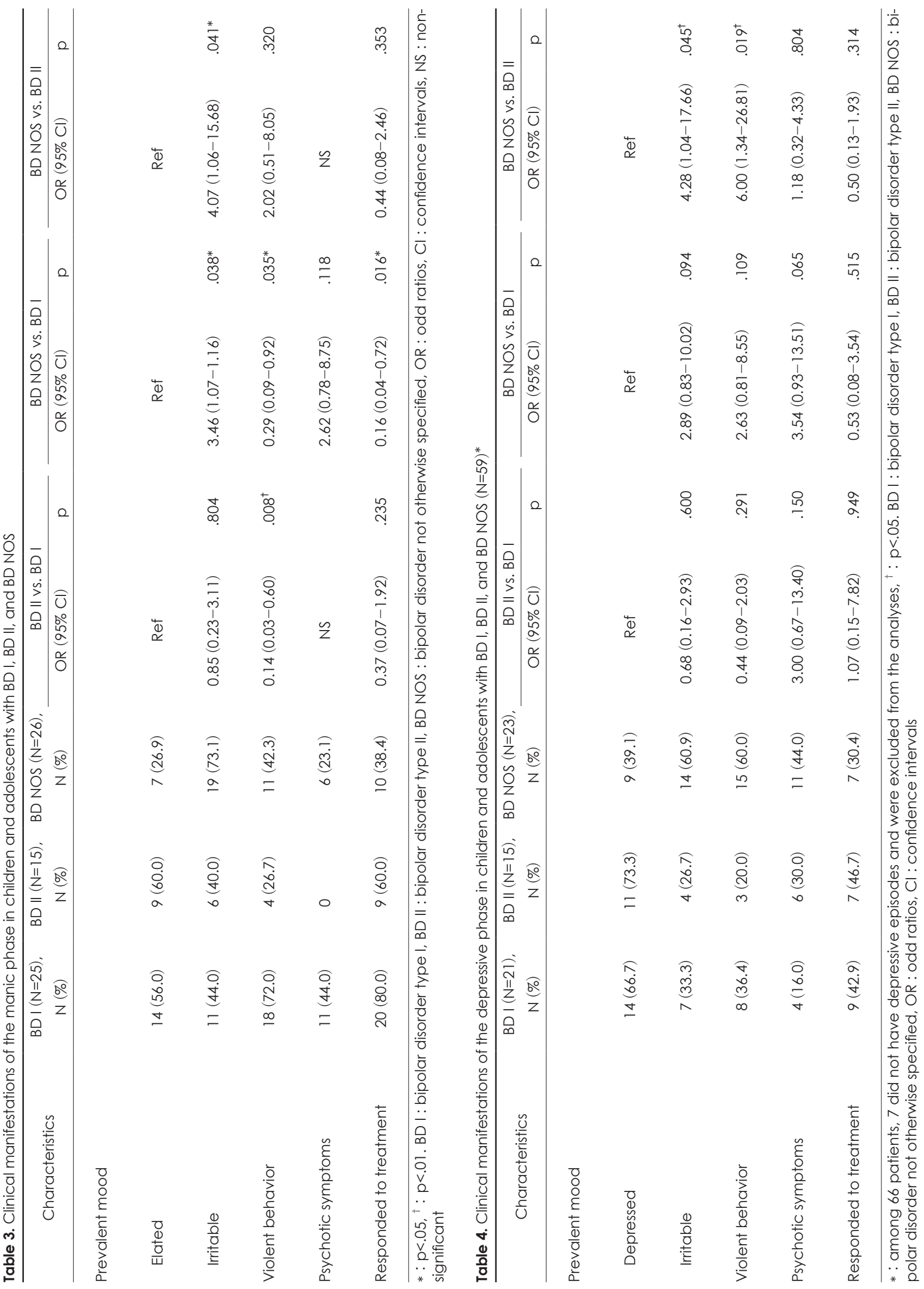




\section{Conclusion}

The present observations highlight the heterogeneous characteristics of BD with regard to clinical manifestation, comorbidity, disease course, and treatment response in Korean children and adolescents. The implications for the usefulness of subtype categories should continue to be investigated.

\section{References}

1) American Psychiatric Association. Diagnostic and Statistical Manual of Mental Disorders: DSM-4. 4th ed. Washington, DC: American Psychiatric Association;1994.

2) Akiskal HS. The prevalent clinical spectrum of bipolar disorders: beyond DSM-IV. J Clin Psychopharmacol 1996;16(2 Suppl 1):4S$14 \mathrm{~S}$.

3) Faedda GL, Baldessarini RJ, Glovinsky IP, Austin NB. Pediatric bipolar disorder: phenomenology and course of illness. Bipolar Disord 2004;6:305-313.

4) Findling RL, Gracious BL, McNamara NK, Youngstrom EA, Demeter CA, Branicky LA, et al. Rapid, continuous cycling and psychiatric co-morbidity in pediatric bipolar I disorder. Bipolar Disord 2001;3:202-210.

5) Geller B, Sun K, Zimerman B, Luby J, Frazier J, Williams M. Complex and rapid-cycling in bipolar children and adolescents: a preliminary study. J Affect Disord 1995;34:259-268.

6) Masi G, Perugi G, Toni C, Millepiedi S, Mucci M, Bertini N, et al. The clinical phenotypes of juvenile bipolar disorder: toward a validation of the episodic-chronic-distinction. Biol Psychiatry 2006; 59:603-610.

7) Carlson GA, Pine DS, Nottelmann E, Leibenluft E. Defining subtypes of childhood bipolar disorder: response and commentary. J Am Acad Child Adolesc Psychiatry 2004;43:3-4.

8) American Psychiatric Association. Diagnostic and Statistical Manual of Mental Disorders: DSM-5. 5th ed. Washington, DC: American Psychiatric Association;2013.
9) Masi G, Perugi G, Millepiedi S, Mucci M, Pari C, Pfanner C, et al. Clinical implications of DSM-IV subtyping of bipolar disorders in referred children and adolescents. J Am Acad Child Adolesc Psychiatry 2007:46:1299-1306.

10) Birmaher B, Axelson D, Strober M, Gill MK, Valeri S, Chiappetta $\mathbf{L}$, et al. Clinical course of children and adolescents with bipolar spectrum disorders. Arch Gen Psychiatry 2006;63:175-183.

11) American Psychiatric Association. Diagnostic and Statistical Manual of Mental Disorders: DSM-4-TR. 4th ed., text revision. Washington, DC: American Psychiatric Association;2000.

12) Park K, Yoon J, Park H. The Manual of Korean Educational Development Institute-Wechsler Intelligence Scale for Children-Revised. Seoul: Korean Educational Development Institute;1991.

13) Bora E, Yucel M, Pantelis C. Cognitive endophenotypes of bipolar disorder: a meta-analysis of neuropsychological deficits in euthymic patients and their first-degree relatives. J Affect Disord 2009; 113:1-20.

14) Joseph MF, Frazier TW, Youngstrom EA, Soares JC. A quantitative and qualitative review of neurocognitive performance in pediatric bipolar disorder. J Child Adolesc Psychopharmacol 2008;18: 595-605.

15) Schenkel LS, West AE, Jacobs R, Sweeney JA, Pavuluri MN. Cognitive dysfunction is worse among pediatric patients with bipolar disorder Type I than Type II. J Child Psychol Psychiatry 2012;53: 775-781.

16) Biederman J, Russell R, Soriano J, Wozniak J, Faraone SV. Clinical features of children with both ADHD and mania: does ascertainment source make a difference? J Affect Disord 1998;51:101112.

17) Sachs GS, Baldassano CF, Truman CJ, Guille C. Comorbidity of attention deficit hyperactivity disorder with early- and late-onset bipolar disorder. Am J Psychiatry 2000;157:466-468.

18) Wozniak J, Spencer T, Biederman J, Kwon A, Monuteaux M, Rettew J, et al. The clinical characteristics of unipolar vs. bipolar major depression in ADHD youth. J Affect Disord 2004;82 Suppl 1:S59-S69.

19) Dilsaver SC, Henderson-Fuller S, Akiskal HS. Occult mood disorders in 104 consecutively presenting children referred for the treatment of attention-deficit/hyperactivity disorder in a community mental health clinic. J Clin Psychiatry 2003;64:1170-1176; quiz, 1274-1276. 